\title{
Metástase orbital como primeira manifestação clínica de hepatocarcinoma
}

\author{
Orbital metastasis as the first clinical manifestation \\ of hepatocellular carcinoma
}

Mariluze Sardinha ${ }^{1}$, Epaminondas de Souza Mendes Junior'리 Juliana Cabral Duarte Brandão ${ }^{3}$, Paulo Roberto Fontes Athanázio ${ }^{4}$, Roberto Lorens Marback ${ }^{5}$

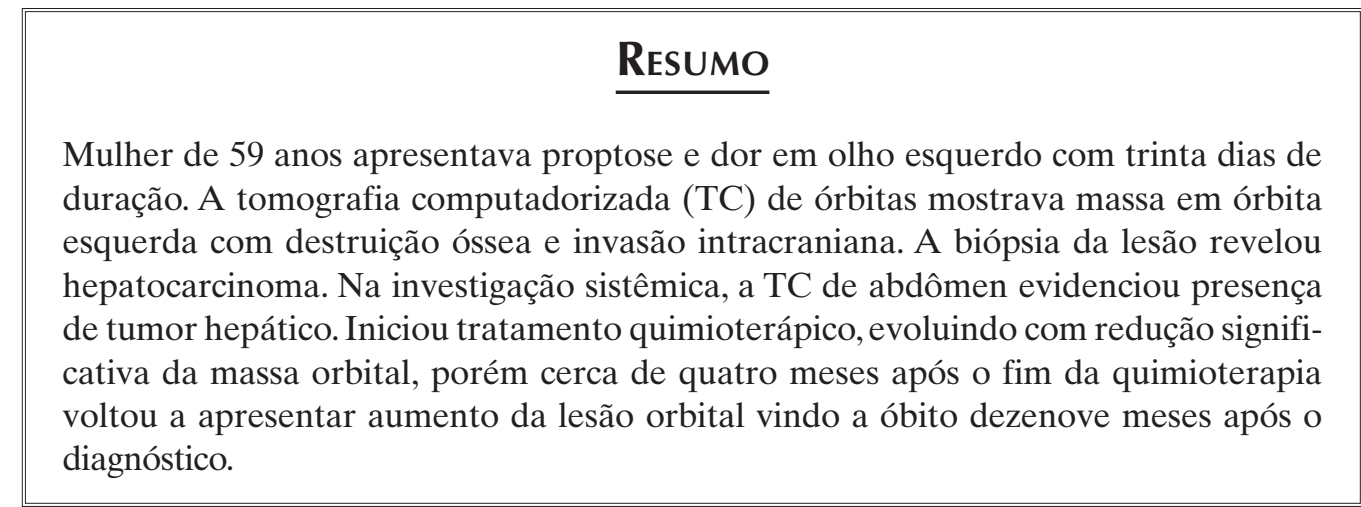

Descritores: Metástase neoplásica/diagnóstico; Metástase neoplásica/patologia; Órbita/patologia; Neoplasias orbitárias/secundário; Carcinoma hepatocelular/diagnóstico; Carcinoma hepatocelular/patologia; Relatos de casos

\footnotetext{
'Doutora, Médica Colaboradora do Setor de Oculoplástica do Serviço de Oftalmologia do Hospital Universitário Professor Edgar Santos da Universidade Federal da Bahia - UFBA - Salvador (BA), Brasil;

²Estagiário em Oculoplástica no Serviço de Oftalmologia do Hospital Universitário Professor Edgar Santos da Universidade Federal da Bahia - UFBA - Salvador (BA), Brasil;

${ }^{3}$ Residente do $3^{\circ}$ ano do Serviço de Anatomia Patológica do Hospital Universitário Professor Edgar Santos da Universidade Federal da Bahia - UFBA - Salvador (BA), Brasil;

${ }^{4}$ Professor Adjunto de Anatomia Patológica da Faculdade de Medicina da Bahia da Universidade Federal da Bahia - UFBA - Salvador (BA), Brasil;

${ }_{5}^{5}$ Professor Titular de Oftalmologia da Faculdade de Medicina da Bahia da Universidade Federal da Bahia - UFBA - Salvador (BA), Brasil.

* Trabalho realizado no Serviço de Oftalmologia do Hospital Universitário Professor Edgar Santos da Universidade Federal da Bahia UFBA - Salvador (BA), Brasil

* Apresentado como Pôster na XX Jornada de Oftalmologia Centro de Estudos Professor Heitor Marback - Salvador (BA) em 22 e 23 de fevereiro de 2008

* Prêmio de Melhor Pôster no Simpósio Nacional de Plástica Ocular, Vias Lacrimais e Órbita da SBCPO (Sociedade Brasileira de Cirurgia Plástica Ocular) - São Paulo (SP) em 11 e 12 de Abril de 2008
}

Recebido para publicação em: 11/6/2009 - Aceito para publicação em 7/8/2009 


\section{INTRODUÇÃO}

A órbita é local pouco frequente de metástase tumoral $^{(1)}$.A ocorrência de doença metastática na órbita varia de um a treze por cento entre todos os tumores orbitários ${ }^{(2)}$.

Os sítios primários mais comuns de metástases orbitais são: mama (em mulheres), pulmão e próstata (em homens) $)^{(3-4)}$. O carcinoma hepático tem como locais mais comuns de metástase os pulmões, nódulos linfáticos, glândulas adrenais e ossos ${ }^{(5)}$. Metástase orbital de carcinoma hepático primário é rara, sendo poucos os casos descritos na literatura ${ }^{(4-23)}$.

Relatamos caso clínico no qual a metástase orbital foi a primeira manifestação clínica de carcinoma primário de fígado.

\section{Relato de caso}

Mulher de 59 anos foi encaminhada para o serviço de plástica ocular do serviço de Oftalmologia do Hospital Universitário Professor Edgar Santos com história de dor em olho esquerdo (OE) há 1 mês. Uma semana antes de procurar atendimento neste serviço observou deslocamento do OE para fora e para baixo (Figura $1 \mathrm{~A})$.
Como antecedentes médicos apresentava hipertensão arterial sistêmica e diabetes mellitus, sob controle medicamentoso. Foi submetida a esplenectomia há trinta e sete anos devido à hipertensão portal esquistossomótica. Realizou biópsia hepática cinco anos após a esplenectomia que revelou fibrose septal e hepatite reacional inespecífica. Apresentava sorologias negativas para hepatites $\mathrm{B}$ e $\mathrm{C}$.

Ao exame, a acuidade visual corrigida era de 20/ 25 em olho direito (OD) e 20/100 em OE. Observou-se à esquerda, proptose com distopia para baixo e limitação da elevação (Figura 1-A). Pressão intraocular (PIO) em $\mathrm{OD}=10 \mathrm{mmHg}$ e $\mathrm{OE}=22 \mathrm{mmHg}$, em uso de colírio combinado de tartarato de brimonidina $0,1 \%$ e maleato de timolol $0,5 \%$. Ao exame de fundo de olho apresentava exsudatos duros em ambos os olhos e no OE também foi observado edema de papila com hemorragia peripapilar.

Tomografia computadorizada (TC) de órbitas mostrava lesão expansiva retrobulbar em órbita esquerda com erosão óssea e extensão intracraniana (Figura 1-B e 1-C). A biópsia da lesão evidenciou neoplasia maligna constituída por células grandes, atípicas, com núcleos volumosos, nucléolos evidentes e arranjo trabecular organóide (Figura 2-A). A imuno-

\section{Quadro 1}

Sumário de 21 casos relatados de hepatocarcinoma metastático para órbita

\begin{tabular}{|c|c|c|c|}
\hline Autor & Idade (anos) & Sexo & Sinais e sintomas \\
\hline Lubin et al. (1980) ${ }^{(6)}$ & 69 & M & Proptose, dor \\
\hline Zubler et al. (1981) (7) & 64 & M & Proptose, BAV, oftalmoplegia \\
\hline Wakisaka et al. (1990) & 58 & M & Proptose, diplopia, ptose \\
\hline Phanthumchinda et al. (1992) & 29 & $\mathrm{~F}$ & Dor, oftalmoplegia \\
\hline Hosokawa et al. (1994) & 70 & M & BAV, cefaléia \\
\hline Kami et al. (1994) ${ }^{(13)}$ & 60 & M & Proptose, cefaléia \\
\hline Loo et al. (1994) ${ }^{(12)}$ & 71 & M & Dor, BAV \\
\hline Schwab et al. (1994) & 19 & M & Proptose, ceratite de exposição \\
\hline Tranfa et al. (1994) ${ }^{(10)}$ & 85 & $\mathrm{M}$ & Proptose, dor, BAV \\
\hline Font et al. (1998) ${ }^{(4)}$ & 79 & $\mathrm{~F}$ & Proptose, dor e BAV \\
\hline Scolyer et al. (1999) ${ }^{(15)}$ & 78 & & Massa periorbital \\
\hline Kim et al. (2000) & 56 & $\mathrm{~F}$ & Estrabismo, dor \\
\hline Barbat et al. (2000) ${ }^{(16)}$ & 57 & $\mathrm{M}$ & Diplopia, BAV \\
\hline Labetoulle et al. (2001) & 84 & M & Proptose \\
\hline Gupta et al. (2005) (19) & 45 & M & Proptose, RMO, massa periorbital \\
\hline Machado-Neto et al. (2006) & 57 & $\mathrm{M}$ & Proptose, massa periorbital \\
\hline Srinivasan et al. (2007) & 76 & $\mathrm{~F}$ & Proptose, BAV, dor \\
\hline Hirunwiwtkul et al. (2008) ${ }^{(5)}$ & 74 & $\mathrm{~F}$ & Dor, proptose, cefaléia, BAV, ptose \\
\hline Pitts et al. (2008) (22) & 6147 & FM & Proptose, BAV e dor \\
\hline Fonseca Junior et al. (2008) & 57 & $\mathrm{M}$ & Proptose, BAV, dor \\
\hline
\end{tabular}

BAV - baixa de acuidade visual; PIO - pressão intraocular; RMO - restrição de movimentação ocular; $\mathrm{M}$ - masculino; F - feminino 
histoquímica mostrou células positivas para hepatócito com anticorpo Hep Par 1 (hepatocyte parafffin 1) (Figura 2B) e negativas para citoqueratinas 7 e 20 . Os aspectos morfológicos e imuno-histoquímicos permitiram o diagnóstico de hepatocarcinoma. TC de abdômen revelou lesão expansiva medindo aproximadamente $6,0 \mathrm{x}$ $3,5 \times 2,5 \mathrm{~cm}$ localizada em segmento IV do fígado (Figura $2 \mathrm{C}$ ).

Foi encaminhada para serviço de Oncologia e retornou após realização de seis sessões de quimioterapia. Evoluiu com melhora da acuidade visual em OE para 20/40, acentuada melhora da proptose, controle da PIO e resolução do edema de papila em OE. A TC de órbitas evidenciava diminuição da massa orbitária, porém, cerca de três meses após o término da quimioterapia, voltou a apresentar aumento da massa orbital. Manteve acompanhamento oncológico e oftalmológico com sobrevida, após o diagnóstico, de um ano e sete meses.

\section{DısCUSSÃO}

Metástases orbitais são menos frequentes que metástases oculares, embora sua frequência venha aumentando. Esse aumento reflete sobrevida mais prolongada de pacientes com câncer ${ }^{(24)}$.

$\mathrm{Na}$ maioria das grandes séries ocidentais publicadas sobre metástase orbital nenhum caso de carcinoma hepático foi relatado ${ }^{(2,3,25)}$. Até o momento, encontramos vinte e um casos de metástase orbital secundária a carcinoma hepático com comprovação histopatológica (Quadro 1) ${ }^{(4-23)}$. Entretanto, em revisão sobre metástases orbitárias, relatada no Japão de 1903 a 1998 , foi observado que dos cento e vinte e oito casos os sítios primários mais frequentes foram pulmão, mama e fígado ${ }^{(26)}$.

Em algumas regiões da África e da Ásia existem uma elevada incidência de carcinoma hepático, estando este entre os principais tipos de câncer e causa de morte no Japão ${ }^{(20,26)}$. Dentre os fatores relacionados ao grande número de casos deste tipo de tumor nessas áreas está a pré-disposição genética associada à prevalência, relativamente alta de cirrose alcoólica, hepatites crônicas B e C e exposição à aflatoxinas ${ }^{(5)}$.

Nossa paciente apresentava sorologia negativa para hepatites $\mathrm{B}$ e C. No que diz respeito à doença hepática, a paciente tinha diagnóstico de hipertensão portal esquistossomótica. Apesar de já ter sido citada a associação entre esquistossomose mansônica e hepatocarcinoma ${ }^{(27)}$, não existe adequada evidência do

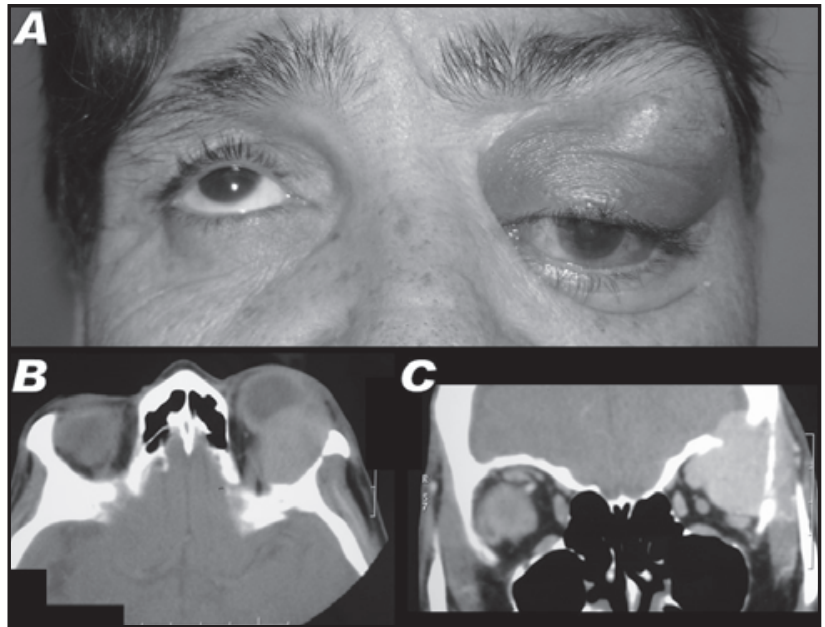

Figura 1A: Proptose, distopia e restrição de elevação em olho esquerdo; B e C: Lesão expansiva retrobulbar em órbita esquerda com erosão óssea e extensão intracraniana

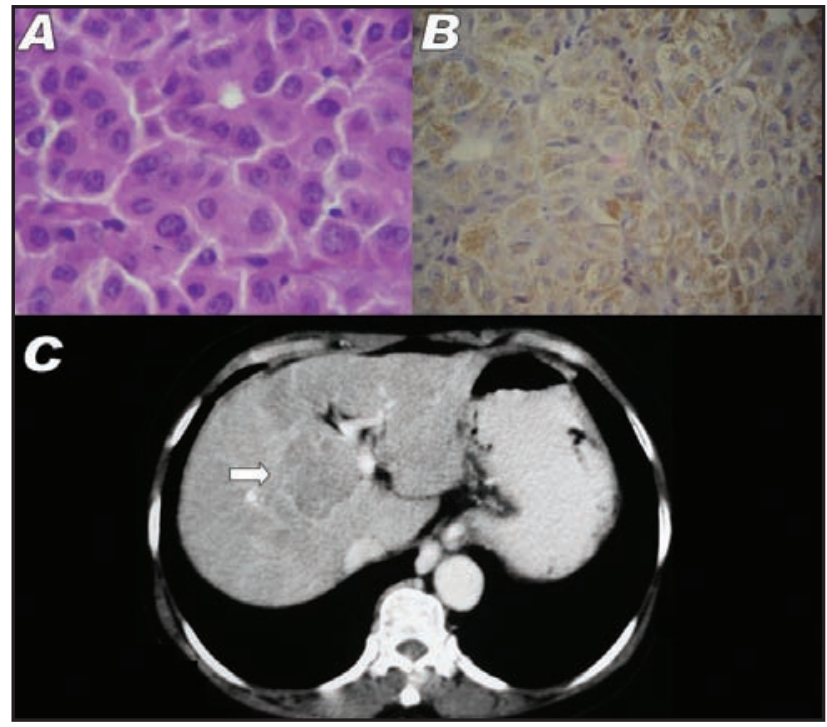

Figura 2A: Aspecto histológico; B: Imunohistoquímica positivo com Hep Par1. C: Lesão expansiva localizada em fígado (seta branca)

potencial carcinogênico desta doença em humanos ${ }^{(28,29)}$.

Os pacientes com metástase orbital secundária a hepatocarcinoma apresentam como manifestações típicas dor, proptose e perda visual ${ }^{(4,26)}$. A maioria dos casos relatados é de pessoas idosas, com idade entre a sétima e oitava década de vida (Quadro 1). A localização mais comum da lesão na órbita é o quadrante superior externo e a destruição óssea é comum ${ }^{(5)}$. Nos relatos de caso e séries descritas não houve predomínio quanto a lateralidade, mas a lesão foi unilateral em todos os casos. Com semelhança ao caso por nós em relato, alguns casos publicados apresentaram a metástase orbital como 
primeira manifestação do carcinoma hepático $(7,16,20,23,26)$.

O diagnóstico de metástase orbital por carcinoma hepático é baseado no exame histopatológico da lesão, o qual se caracteriza por um padrão trabecular, associado com grandes células poligonais que têm um citoplasma acidófilo, grandes núcleos ovais ou redondos e nucléolos proeminentes ${ }^{(19,20)}$. Estudo imuno-histoquímico pode ajudar no diagnóstico, sendo os principais marcadores CAM 5.2,AE1/AE3, AFP, CEA ${ }^{(5,20)}$ e Hep Par 1. Este é considerado um marcador muito sensível e específico para hepatócitos normais e neoplásicos tendo sido usado no diagnóstico de carcinoma hepatocelular ${ }^{(30,31)}$.

O tratamento para hepatocarcinoma metastático tem eficácia limitada. No caso de metástase orbital, o tratamento mais freqüente é radioterapia focal. Alguns pacientes também têm sido tratados com quimioterapia sistêmica, como em nosso caso. A cirurgia está indicada basicamente para fins diagnósticos. Devido à realização da quimioterapia em outro Serviço, não tivemos acesso ao tipo de tratamento utilizado. O prognóstico dos pacientes com metástase orbital por hepatocarcinoma é bastante reservado, com sobrevida média de 10.4 meses $^{(26)}$. Nossa paciente apresentou redução da massa orbital após a quimioterapia. Porém, três meses após o tratamento, voltou a apresentar crescimento da lesão orbital evoluindo para o óbito um ano e sete meses após o diagnóstico.

Embora raro, o hepatocarcinoma deve ser considerado no diagnóstico diferencial de metástase orbital com sítio primário desconhecido, sobretudo em pacientes com fatores de risco para este tipo de tumor.

\section{Abstract}

A fifty nine years old female presented a painful proptotic left eye for thirty days. Computed thomography of the orbits disclosed an orbital mass associated with osteolysis and intracranial invasion. Biopsy of the orbital tumor revealed the diagnosis of hepatocellular carcinoma. Computed tomography showed the liver as the primary site of the tumor. Chemoterapy induced a significativ reduction of the orbital tumor. After four months, the orbital tumor increased its size and the pacient died ninetheen months after the diagnosis.

Keywords: Neoplasm metastasis/diagnosis; Neoplasm metastasis/pathology; Orbit/pathology;Orbital neoplasms/secondary; Carcinoma, hepatocellular/ diagnosis; Carcinoma, hepatocellular/pathology

\section{RefERÊNCIAS}

1. Shields JA, Shields CL, Scartozzi R. Survey of 1264 patients with orbital tumors and simulating lesions: The 2002 Montgomery Lecture, part 1. Ophthalmology. 2004;111(5):9971008.

2. Shields JA, Shields CL, Brotman HK, Carvalho C, Perez N, Eagle RC Jr. Cancer metastatic to the orbit. The 2000 Robert M. Curts Lecture. Ophthal Plast Reconstr Surg. 2001;17(5):346-54.

3. Char DH, Miller T, Kroll S. Orbital metastasis: diagnosis and course. Br J Ophthalmol. 1997;81(5):386-90;

4. Font RL, Maturi RK, Small RG, Garcia-Rojas M. Hepatocellular carcinoma metastatic to the orbit. Arch Ophthalmol. 1998;116(7):942-5;

5. Hirunwiwatkul P, Tirakunwichcha S, Meesuaypong $\mathrm{P}$, Shuangshoti S.Orbital metastasis of hepatocellular carcinoma. J Neuroophthalmol. 2008;28(1):47-51.

6. Lubin JR, Grove AS, Zakov ZN, Albert DM. Hepatoma metastatic to the orbit. Am J Ophthalmol. 1980;89(2):268-73.

7. Zubler MA, Rivera R, Lane M. Hepatoma presenting as a retro-orbital metastasis. Cancer. 1981;48(8):1883-5.

8. Wakisaka S, Tashiro M, Nakano S, Kita T, Kisanuki H, Kinoshita

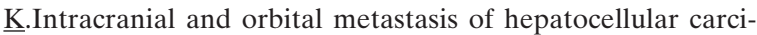
noma: report of two cases. Neurosurgery. 1990;26(5):863-6.

9. Phanthumchinda K, Hemachuda T. Superior orbital fissure syndrome as a presenting symptom in hepatocellular carcinoma. J Med Assoc Thai. 1992;75(1):62-5.

10. Tranfa F, Cennamo G, Rosa N, Boscaino A, Bonavolontà G. An unusual orbital lesion: hepatoma metastatic to the orbit. Ophthalmologica. 1994;208(6):329-32.

11. Schwab L, Doshi H, Shields JA, Kagame K, Chana H. Hepatocellular carcinoma metastatic to the orbit in an African patient. Ophthalmic Surg. 1994;25(2):105-6.

12. Loo KT, Tsui WM, Chung KH, Ho LC, Tang SK, Tse CH.Hepatocellular carcinoma metastasizing to the brain and orbit: report of three cases. Pathology. 1994;26(2):119-22.

13. Kami H, Wada M, Matsuura T, Araya S, Yamashita M, Shikano $\underline{\text { S}}$,et al. [Case of hepatocellular carcinoma with an orbital metastasis as the initial symptom]. Nippon Naika Gakkai Zasshi. 1994;83(4):622-4. Japanese.

14. Hosokawa C, Kawabe J, Okamura T, Kamino T, Ikeda H, Ochi $\underline{\mathrm{H}}$, et al. Usefulness of $99 \mathrm{mTc}-\mathrm{PMT}$ SPECT and 18F-FDG PET in diagnosing orbital metastasis of hepatocellular carcinoma. Kaku Igaku. 1994;31(10):1237-42. Japanese.

15. Scolyer RA, Painter DM, Harper CG, Lee CS. Hepatocellular carcinoma metastasizing to the orbit diagnosed by fine needle aspiration cytology. Pathology. 1999;31(4):350-3.

16. Barbat V, Morin Y, Metge F, Hamard H.[Hepatocellular carcinoma: a case revealed by metastatic orbital tumor]. Rev Med Interne. 2000 Jan;21(1):86-90. French.

17. Kim IT, Na SC, Jung BY. Hepatocellular carcinoma metastatic to the orbit. Korean J Ophthalmol. 2000;14(2):97-102.

18. Labetoulle M, Capelli C, Dubreuil F, Kirsch O, Perlemuter G, Buffet C,et al. [Orbital metastasis of hepatocellular carcinoma]. Gastroenterol Clin Biol. 2001;25(10):914-5. French.

19. Gupta R, Honavar SG, Vemuganti GK. Orbital metastasis from hepatocellular carcinoma. Surv Ophthalmol. 2005;50(5):485-9.

20. Machado-Netto MC, Lacerda ECA, Heinke T, et al. Massive orbital metastasis of hepatocellular carcinoma. Clinics. 2006;61(4):359-62. 
21. Srinivasan R, Krishnanand G. Cytologic diagnosis of metastatic hepatocellular carcinoma presenting as an orbital mass. A case report. Acta Cytol. 2007;51(1):83-5.

22. Pitts J, Chang CH, Mavrikakis I, Shaikh A, Rootman J. Hepatocellular carcinoma presenting as orbital bone metastasis. Ophthal Plast Reconstr Surg. 2008;24(6):477-9.

23. Fonseca Júnior NL, Frizon L, Paves L, Wolosker AMB, Manso PG. Metástase orbitária como único achado em paciente com hepatocarcinoma: relato de caso. Arq Bras Oftalmol. 2008;71(6):865-7.

24. Rootman J. Orbital metastases. In: Pine J, Field C, editors. Diseases of the orbit: a multidisciplinary approach. Philadelphia: Lippincott Williams and Wilkins, 2003 . p. 330-9.

25. Ferry AP, Font RL. Carcinoma metastatic to the orbit I. A clinicopathological study of 227 cases. Arch Ophthalmol. 1974;92(4):276-86.

26. Amemiya T, Hayashida H, Dake Y. Metastatic orbital tumors in Japan: a review of the literature. Ophthalmic Epidemiol. 2002;9(1):35-47.

27. Mott KE. Possible relationship of Schistosoma infection and liver carcinoma. Trans R Soc Trop Med Hyg. 1978;725(5):552-3.
28. Pereira FE, Gonçalves CS. The association of Schistosoma mansoni infection with hepatocellular carcinoma. Rev Soc Bras Med Trop. 1984; 17(1):73-64.

29. Yosry A. Schistosomiasis and neoplasia. Contrib Microbiol. 2006;13:81-100.

30. Kanitakis J, Chouvet B, Claudy A, Scoazec JY. Immunoreactivity of hepatocyte paraffin 1 monoclonal antibody in cutaneous metastático tumors. Am J Clin Pathol. 2004;122(1):85-9.

31. Amarapurkar AD, Rege JD, Joshi AS, Vaiphei K, Amarapurkar DN. Utilization of antihepatocyte clone OCH1E5 (Hep Par 1) in histological evaluation of liver tumors. Indian J Pathol Microbiol. 2006;49(3):341-4.

\section{Endereço para correspondência:}

Epaminondas de Souza Mendes Junior

Rua Silveira Martins, Cond. Pomar do Cabula, Edf

Vivenda das Orquídeas, no 267, Apto 408, Cabula,

CEP 41150-000 - Salvador - BA

Email: epamjr@superig.com.br 